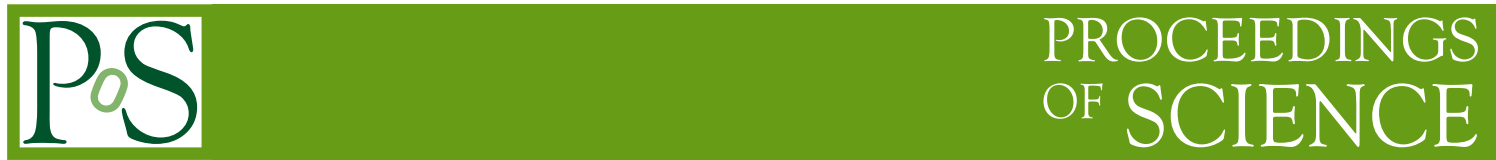

\title{
Diboson Production at the Tevatron
}

\section{Jadranka Sekaric*}

University of Kansas for the CDF and DØ Collaborations

E-mail: sekaric@fnal.gov

Here we summarize the recent measurements of the diboson production cross sections and limits on trilinear gauge boson couplings using $1-5 \mathrm{fb}^{-1}$ of Tevatron data collected by the CDF and DØ detectors. These results are the most precise to date from a hadron collider.

XVIII International Workshop on Deep-Inelastic Scattering and Related Subjects, DIS 2010

April 19-23, 2010

Firenze, Italy

*Speaker. 


\section{Diboson Production}

Precision measurements of diboson processes play an important role in electroweak physics and searches for New Physics (NP) which may exist at some energy scale $\Lambda$. NP searches are often related to measurements of the diboson production cross sections and the trilinear gauge boson couplings (TGCs) [1]. In presence of NP these observables are expected to deviate from their SM predictions. The charged TGCs studied in $W W, W Z$ and $W \gamma$ production are $\Delta g_{1}^{V}-\Delta \kappa_{V}-\lambda_{V}$ where $V=Z$ or $\gamma$, and $\Delta$ represents the deviation from the SM prediction. In the SM, $\Delta g_{1}^{V}=\Delta \kappa_{V}=\lambda_{V}=$ 0 . The neutral TGCs $h_{i}^{V}(i=3,4)$ studied in $Z \gamma$ production are not allowed in the SM and their values are predicted to be zero. Diboson production is an important background in studies of the top quark, and searches for the Higgs boson and SUSY particles. Thus, precise knowledge of diboson processes and their proper modeling is important for current and future studies.

\section{2. $Z \gamma$ Production}

The $e^{+} e^{-} \gamma\left(\mu^{+} \mu^{-} \gamma\right)$ events selected from $1 \mathrm{fb}^{-1}\left(2 \mathrm{fb}^{-1}\right)$ of CDF data require a photon with transverse energy $E_{T}>7 \mathrm{GeV}$, spatially separated from a lepton by $\Delta R_{l \gamma}>0.7$. The three-body mass cut of $100 \mathrm{GeV}$ separates events which originate from two different processes, initial and final state radiation (ISR and FSR), and their individual cross section measurements. The combined ISR+FSR cross section is measured to be $\sigma_{Z \gamma \rightarrow l \bar{l} \gamma}=4.6 \pm 0.4_{(\text {stat }+ \text { syst })} \pm 0.3_{(\text {lumi })}$ pb [2]. Photon $E_{T}$ spectra from $l \bar{l} \gamma$ events shown in Fig. 1 are combined with $v \bar{v} \gamma$ candidate events selected from $2 \mathrm{fb}^{-1}$ of data and used to set the limits on TGCs. The one-dimensional 95\% C.L. limits on $h_{3,4}^{\gamma, Z}$ at $\Lambda=1.2 \mathrm{TeV}$ are $\left|h_{3}^{\gamma}\right|<0.051,\left|h_{3}^{Z}\right|<0.050$ and $\left|h_{4}^{\gamma, Z}\right|<0.0034$. At the DØ experiment the $v \bar{v} \gamma$ events are reconstructed from $3.6 \mathrm{fb}^{-1}$ of data requiring one isolated photon of $E_{T}>90 \mathrm{GeV}$ and missing transverse energy of $\mathbb{E}_{T}>70 \mathrm{GeV}$. The cross section is measured to be $\sigma_{Z \gamma \rightarrow v \bar{v} \gamma}=$ $32 \pm 9_{(\text {stat }+ \text { syst })} \pm 2_{(\text {lumi })} \mathrm{fb}$ [3] with a observed signal significance of 5.1 standard deviations (s.d.). The photon $E_{T}$ spectrum is used to set limits on $Z \gamma \gamma / Z Z \gamma$ TGCs. The one-dimensional 95\% C.L limits at $\Lambda=1.5 \mathrm{TeV}$ are $\left|h_{30}^{\gamma}\right|<0.036,\left|h_{30}^{Z}\right|<0.035$ and $\left|h_{40}^{\gamma, Z}\right|<0.0019$ [3]. The combination with a $1 \mathrm{fb}^{-1} l \bar{l} \gamma$ analysis [4] results in the most restrictive limits on these couplings of $\left|h_{30}^{\gamma, Z}\right|<$ 0.033 and $\left|h_{40}^{\gamma, Z}\right|<0.0017$ of which three of them $\left(h_{40}^{\gamma, Z}\right.$ and $\left.h_{30}^{Z}\right)$ are currently world bests. The $Z \gamma$ cross sections measured by the two collaborations are in agreement with the next-to-leading (NLO) prediction [5].

\section{ZZ Production}

Three $l \bar{l} l^{\prime} \bar{l}^{\prime}\left(l, l^{\prime}=e, \mu\right)$ candidate events were observed in $1.7 \mathrm{fb}^{-1}$ of $\mathrm{D} \varnothing$ data. This was the first observation of $Z Z$ production at the hadron collider with a significance of 5.3 s.d. [6]. The measured cross section is $\sigma_{Z Z}=1.75_{-0.87}^{+1.28}($ stat + syst $) \mathrm{pb}$. The $l \bar{l} \nu \bar{v}(l=e, \mu)$ events selected from $2.7 \mathrm{fb}^{-1}$ of $\mathrm{D} \varnothing$ data were used to measure the cross section of $\sigma_{Z Z}=2.01 \pm 0.97_{\text {(stat }+ \text { syst })} \mathrm{pb}$ [7]. The combination of all analyzed $Z Z$ final states results in measured cross section of $\sigma_{Z Z}=1.60 \pm$ $0.65_{\text {(stat }+ \text { syst) }} \mathrm{pb}$ and a significance of 5.7 s.d.. Five $l \bar{l} l^{\prime} \bar{l}^{\prime}$ candidate events were selected in $4.8 \mathrm{fb}^{-1}$ of CDF data with significance of 5.7 s.d. measuring the cross section of $\sigma_{Z Z}=1.56_{-0.68}^{+0.84}$ (stat + syst) pb. All measured ZZ cross sections are in agreement with the NLO prediction [8]. 


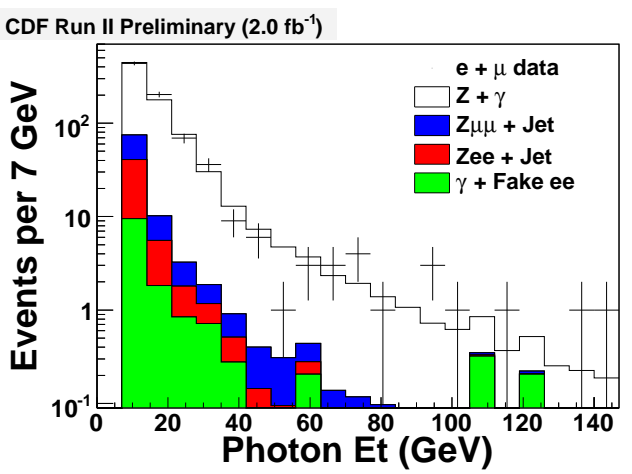

Figure 1: Photon $E_{T}$ spectra of $Z \gamma \rightarrow l \bar{l} \gamma$ candidate events selected from CDF data.

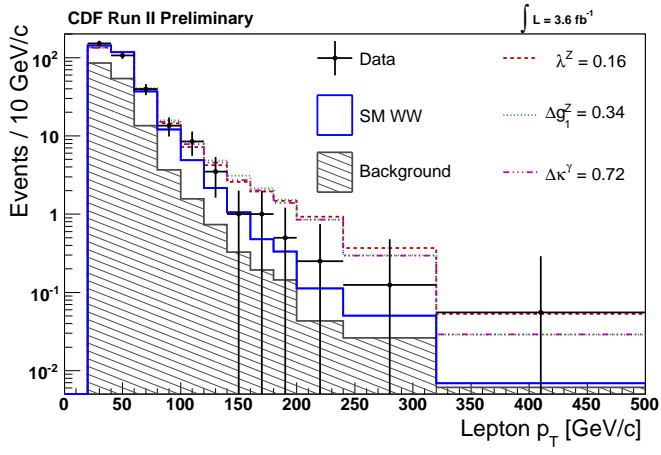

Figure 2: $\quad$ Leading lepton $p_{T}$ distribution compared to the SM and different TGC predictions.

\section{4. $W W$ Production in Leptonic Final States}

The most precise $W W$ cross section measurements at a hadron collider were performed analyzing $l v l^{\prime} v\left(l, l^{\prime}=e, \mu\right)$ final states with $3.6 \mathrm{fb}^{-1}$ of $\mathrm{CDF}$ data [9] and $1.0 \mathrm{fb}^{-1}$ of D $\varnothing$ data [10]. Both event selections require the presence of two oppositely charged leptons and significant $\mathbb{E}_{T}$ per event. After all selection criteria were applied, 654 candidate events were found in CDF data yielding the cross section of $\sigma_{W W}=12.1_{-1.66}^{+1.84}($ stat + syst $) \mathrm{pb}$. The lepton $p_{T}$ spectrum shown in Fig. 2 has been used to set the one-dimensional 95\% C.L. limits on TGCs for $\Lambda=2 \mathrm{TeV}$ of $-0.57<\Delta \kappa_{\gamma}<0.65,-0.14<\lambda_{\gamma}=\lambda_{Z}<0.15$ and $-0.22<\Delta g_{1}^{Z}<0.30$ under the $S U(2)_{L} \times U(1)_{Y^{-}}$ conserving constraints [11]. The $\mathrm{D} \emptyset$ experiment selected 100 candidate events and measured the cross section of $\sigma_{W W}=11.5 \pm 2.1_{(\text {stat }+ \text { syst })} \pm 0.7_{(\text {lumi })} \mathrm{pb}$. The $p_{T}$ distributions of the leading and trailing leptons were used to set limits on anomalous TGCs. The one-dimensional 95\% C.L. limits for $\Lambda=2 \mathrm{TeV}$ are $-0.54<\Delta \kappa_{\gamma}<0.83,-0.14<\lambda_{\gamma}=\lambda_{Z}<0.18$ and $-0.14<\Delta g_{1}^{Z}<0.30$ under the $S U(2)_{L} \times U(1)_{Y}$-conserving constraints, and $-0.12<\Delta \kappa_{\gamma}=\Delta \kappa_{Z}<0.35$ and $-0.14<\lambda_{\gamma}=$ $\lambda_{Z}<0.18$ under the assumption that $\gamma W W$ and $Z W W$ couplings are equal [1]. Both measurements are in agreement with the SM NLO prediction of $12.4 \pm 0.8 \mathrm{pb}$ [8].

\section{5. $W W$ and $W Z$ Production in Dijet Final States}

The first observation of vector boson pair production in a dijet final states using $3.5 \mathrm{fb}^{-1}$ of data has been presented by the CDF Collaboration [12]. The analysis is based on selecting events with $E_{T}>60 \mathrm{GeV}$, and two jets with $p_{T}>25 \mathrm{GeV}$, without an explicit requirement on the charged lepton. The measured production cross section of $\sigma_{W W+W Z+Z Z}=18.0 \pm 3.7_{(\text {stat }+ \text { syst })} \pm 1.1_{(\text {lumi })} \mathrm{pb}$ comes from a fit in the dijet mass distribution in the $40 \mathrm{GeV}<\mathrm{M}_{J J}<160 \mathrm{GeV}$ mass range (Fig. 3) with a significance of 5.3 s.d. Imposing an explicit requirement on the charged lepton and adding more data, the CDF experiment measures $\sigma_{W W+W Z}$ with two different methods. The first method is a dijet mass fit in $28 \mathrm{GeV}<\mathrm{M}_{J J}<200 \mathrm{GeV}$ mass range in $4.3 \mathrm{fb}^{-1}$ of data [13], with a result of $18.1 \pm 4.1_{(\text {stat }+ \text { syst })}$ pb with a significance of 5.2 s.d.. The second method uses a matrix element-based event probability discriminant to differentiate between signal and background. The 
$W W+W Z$ cross section is then measured to be $16.5_{-3.0}^{+3.3}($ stat + syst $)$ pb with significance of 5.4 s.d. [14].

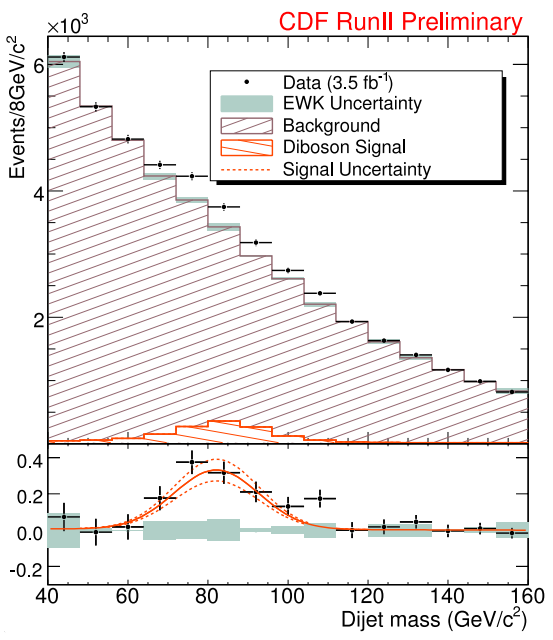

Figure 3: Top: Data-to-background comparison. Bottom: Signal-tobackground-subtracted data comparison. The dashed lines represent the $\pm 1 \sigma$ stat. variations on the extracted signal. The gray band represents the systematic uncertainty due to the electroweak background shape.

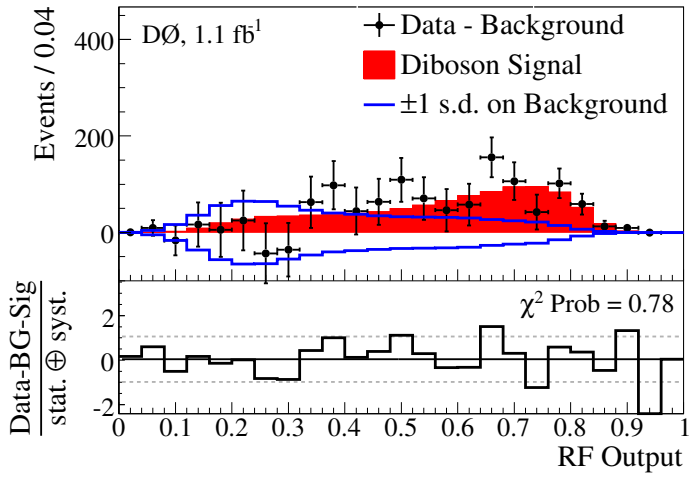

Figure 4: The RF output distribution from the combined $l v q \bar{q}$ channels for data and MC predictions. Top: A comparison of the extracted signal to background-subtracted data, along with $\pm 1 \sigma$ systematic uncertainties on the background. Bottom: The residual distance between the data and signal, divided by the total uncertainty.

The first evidence of $W W+W Z$ production in semi-leptonic final states in $1.1 \mathrm{fb}^{-1}$ of $\mathrm{D} \emptyset$ data yields a cross section of $\sigma_{W W+W Z}=20.2 \pm 4.4_{(\text {stat }+ \text { syst })} \pm 1.2_{(\text {lumi })} \mathrm{pb}$ [15] The cross section is measured from the Random Forest (RF) output distribution shown in Fig. 4. Further, the dijet $p_{T}$ distribution is used to set limits on anomalous TGCs [16]. The one-dimensional 95\% C.L. limits for $\Lambda=2 \mathrm{TeV}$ are $-0.44<\Delta \kappa_{\gamma}<0.55,-0.10<\lambda_{\gamma}=\lambda_{Z}<0.11$ and $-0.12<\Delta g_{1}^{Z}<0.20$ under the $S U(2)_{L} \times U(1)_{Y}$-conserving constraints, and $-0.16<\Delta \kappa_{\gamma}=\Delta \kappa_{Z}<0.23$ and $-0.11<\lambda_{\gamma}=$ $\lambda_{Z}<0.11$ under the $\gamma W W=Z W W$ assumption.

\section{Combined Limits on TGCs}

The $\mathrm{D} \emptyset$ experiment combines four different $0.7-1 \mathrm{fb}^{-1}$ diboson analyses $\left(W W \rightarrow l v l^{\prime} v, W Z \rightarrow\right.$ $l v l \bar{l}$ [17], $W \gamma \rightarrow l v \gamma$ [18] and $W W+W Z \rightarrow l v q \bar{q})$ to set the limits on charged TGCs [19]. Two different relations between anomalous TGCs has been considered for $\Lambda_{N P}=2 \mathrm{TeV}$. Combined one-dimensional $95 \%$ C.L. limits are $-0.29<\Delta \kappa_{\gamma}<0.38,-0.08<\lambda_{\gamma}=\lambda_{Z}<0.08$ and $-0.07<$ $\Delta g_{1}^{Z}<0.16$ under the $S U(2)_{L} \times U(1)_{Y}$-conserving constraints, and $-0.11<\Delta \kappa_{\gamma}=\Delta \kappa_{Z}<0.18$ and $-0.08<\lambda_{\gamma}=\lambda_{Z}<0.08$ under the $\gamma W W=Z W W$ assumption. These are the tightest limits to date on charged TGCs at a hadron collider with sensitivity comparable to that of an individual LEP2 experiment. The measured values and the one-dimensional $68 \%$ C.L. intervals of the $W$ boson magnetic dipole and electric quadrupole moments respecting $S U(2)_{L} \otimes U(1)_{Y}$ symmetry 
with $g_{1}^{Z}=1$ are $\mu_{W}=2.02_{-0.09}^{+0.08}\left(e / 2 M_{W}\right)$ and $q_{W}=-1.00 \pm 0.09\left(e / M_{W}^{2}\right)$, respectively. These are the most stringent results of $\mu_{W}$ and $q_{W}$ moments to date.

\section{Summary}

The CDF and DØ Collaborations have presented recent results of diboson production studies at the Tevatron. Measured cross sections and TGCs are in agreement with the SM predictions. The CDF experiment reports the first observation of the vector boson pair production in dijet final states. The $\mathrm{D} \varnothing$ experiment reports the first observation of $Z \gamma$ production in $v \bar{v} \gamma$ final states, sets the world's tightest limits on the $h_{40}^{\gamma}, h_{40}^{Z}$ and $h_{30}^{Z}$ TGCs, and tightest limits on charged couplings from the combination of four analyses. The $\mathrm{D} \emptyset$ experiment also presents the world's best results on the $W$ boson magnetic dipole and electromagnetic quadrupole moments.

\section{References}

[1] K. Hagiwara, J. Woodside, and D. Zeppenfeld, Phys. Rev. D 41, 2113 (1990); U. Baur, and E. Berger, Phys. Rev. D 47, 4889 (1993).

[2] The CDF Collaboration: T. Aaltonen et al, FERMILAB-PUB-10-073-E (2010).

[3] The DØ Collaboration: V. M. Abazov et al, Phys. Rev. Lett. 102, 201802 (2009).

[4] The DØ Collaboration: V. M. Abazov et al, Phys. Lett. B 653, 378 (2007).

[5] U. Baur, T. Han, and J. Ohnemus, Phys. Rev. D 57, 2823 (1998).

[6] The DØ Collaboration: V. M. Abazov et al, Phys. Rev. Lett. 101, 171803 (2008).

[7] The DØ Collaboration: V. M. Abazov et al, Phys. Rev. D 78, 072002 (2008).

[8] M. Campbell, and R. K. Ellis, Phys. Rev. D 60, 113006 (1999). Cross sections were calculated with the same parameter values given in the paper, except with $\sqrt{s}=1.96 \mathrm{TeV}$.

[9] The CDF Collaboration: T. Aaltonen et al, Phys. Rev. Lett. 104, 201801 (2010).

[10] The DØ Collaboration: V. M. Abazov et al, Phys. Rev. D 78, 072002 (2008).

[11] M. Bilenky, J. L. Kneur, F. M. Renard, and D. Schildknecht, Nucl. Phys. B 409, 22 (1993).

[12] The CDF Collaboration: T. Aaltonen et al, Phys. Rev. Lett. 103, 091803 (2009).

[13] http://www-cdf.fnal.gov/physics/ewk/2010/WW_WZ/index.html

[14] http://www-cdf.fnal.gov/physics/new/hdg//Results_files/results/wwwz_oct09/

[15] The DØ Collaboration: V. M. Abazov et al, Phys. Rev. Lett. 102, 161801 (2009).

[16] The DØ Collaboration: V. M. Abazov et al, Phys. Rev. D 80, 053012 (2009).

[17] The DØ Collaboration: V. M. Abazov et al, Phys. Rev. D 76, 111104(R) (2007).

[18] The DØ Collaboration: V. M. Abazov et al, Phys. Rev. Lett. 100, 241805 (2008).

[19] The DØ Collaboration: V. M. Abazov et al, FERMILAB-PUB-09/380-E (2009). 